\title{
Computation and Visualization of Musical Structures in Chord-Based Simplicial Complexes
}

\author{
Louis Bigo $^{1,2}$, Moreno Andreatta ${ }^{2}$, Jean-Louis Giavitto ${ }^{2}$, \\ Olivier Michel ${ }^{1}$, and Antoine Spicher ${ }^{1}$ \\ 1 LACL/Université Paris-Est Creteil \\ louis.bigo@lacl.fr, \\ \{antoine.spicher, olivier.michel\}@u-pec.fr \\ 2 UMR CNRS STMS 9912/IRCAM \\ \{louis.bigo, jean-louis.giavitto, moreno.andreatta\}@ircam.fr
}

\begin{abstract}
We represent chord collections by simplicial complexes. A temporal organization of the chords corresponds to a path in the complex. A set of $n$-note chords equivalent up to transposition and inversion is represented by a complex related by its 1-skeleton to a generalized Tonnetz. Complexes are computed with MGS, a spatial computing language, and analyzed and visualized in Hexachord, a computer-aided music analysis environment. We introduce the notion of compliance, a measure of the ability of a chord-based simplicial complex to represent a musical object compactly. Some examples illustrate the use of this notion to characterize musical pieces and styles.
\end{abstract}

Keywords: MGS, simplicial complexes, generalized Tonnetze, compliance, Hexachord, chord spaces.

\section{Introduction}

Musical objects and their properties are often represented by spatial structures to understand their algebraic nature, and to study compositional strategies. The spiral array [1], the Tonnetz [2] and orbifolds [3,4] are examples of such spaces. Among their numerous properties, they are respectively well adapted to determine key boundaries, to represent neo-Riemannian operations and voice-leading motions. Most of these spaces (the spiral array includes triangles) propose to represent pitches or chords by points in graph representations.

In this study, we propose to introduce elements of higher dimension than vertices and edges. We represent $n$-note chords by simplices of dimension $(n-1)$ and chord collections by simplicial complexes. The faces of a simplex represent all sub-chords contained in the chord. The dimension enables more specific neighborhood relationship between chords and induces more expressiveness in the chord space. Simplicial complexes are computed with MGS [5], a domain specific programming language dedicated to spatial computing.

Section 2 provides a short introduction to MGS and simplicial complexes. In section 3 we present a method to represent collections of chords by simplicial complexes. These collections are either temporal chord sequences, or chord

J. Yust, J. Wild, and J.A. Burgoyne (Eds.): MCM 2013, LNAI 7937, pp. 38-51, 2013.

(C) Springer-Verlag Berlin Heidelberg 2013 
classes defined by an algebraic property. We show how these last complexes are related to the generalized Tonnetze. In the last section, we present some methods for the visualization of musical sequences in these complexes with Hexachord, a computer-aided music analysis environment. Finally, we introduce the compliance, a measure of the capacity of a complex to represent musical pieces or musical styles.

\section{Technical Background}

MGS. MGS is an experimental domain specific language dedicated to spatial computing, see $[5,6]$. MGS concepts are based on well established notions in algebraic topology [7] and relies on the use of rules to compute declaratively spatial data structures.

In MGS, all data structures are unified under the notion of topological collection. Simplicial complexes defined below are an example of topological collections. Transformations of topological collections are defined by rewriting rules [8] specifying the replacement of sub-collections that can be recursively performed to build new spaces.

Simplicial Complexes. A simplicial complex is a space built by gluing together more elementary spaces called simplices. In this work, simplices are glued using a self-assembly process described below. A simplex (more precisely a $p$-simplex) is the abstraction of a space of dimension $p$. A 0 -simplex corresponds to a point, a 1-simplex corresponds to an edge, a 2-simplex is a triangle, etc. These objects are often represented geometrically as the convex hull of their vertices as shown in Figure 1 for $p$-simplices with $p \in\{0,1,2\}$.

A simplicial d-complex is a simplicial complex where the largest dimension of any simplex is $d$. A graph is simplicial 1-complex. Figure 2 shows a simplicial 2-complex.

For any natural integer $n$, the $n$-skeleton of the simplicial complex $\mathcal{C}$ is defined by the sub-complex $\mathcal{S}$ of $\mathcal{C}$ formed by its simplices of dimension $n$ or less.

A $(p, q)$-path is a sequence of $p$-simplices such that two consecutive simplices are glued to a same $q$-simplex. For example, the usual notion of path in a graph (a sequence of vertices such that from each of its vertices there is an edge to the next vertex in the sequence) corresponds to the notion of $(0,1)$-path.

The $f$-vector of the simplicial $d$-complex $C$ is the sequence $\left(f_{0}, f_{1}, \ldots, f_{d+1}\right)$ where $f_{i}$ is the number of $(i-1)$-simplices of $C$ (by convention, $f_{0}=1$ unless $C$ is the empty complex). For example, the $f$-vector of the complex at the bottom right of Figure 2 is $(1,7,11,5)$.

Self-assembly Process. A simplicial complex can be built from a set of simplices by applying an accretive growing process [9]. The growth process is based on the identification of the simplices boundaries. Nevertheless, this topological operation is not elementary and holds in all dimensions. Figure 1 illustrates the process. First, nodes $A$ and $B$ are merged. Then, the resulting edges $\{A, B\}$ are merged. 


\section{Chord Collections Represented as Simplicial Complexes}

\subsection{Chord Sequences}

We use a method presented in [10] to represent chords as simplices. An $n$-note chord is represented by a $(n-1)$-simplex. In the simplicial representation of chord, a 0-simplex represents a single pitch class. This method requires some abstraction on the chord since some information, as its octave or its duration, are not represented. So from a chord we obtain a set of $n$ pitch classes and then an $(n-1)$-simplex. The simplicial representation of a chord collection is built by:

1. representing each chord of the collection by a simplex as described above.

2. applying the self-assembly process to the resulting collection of simplices.

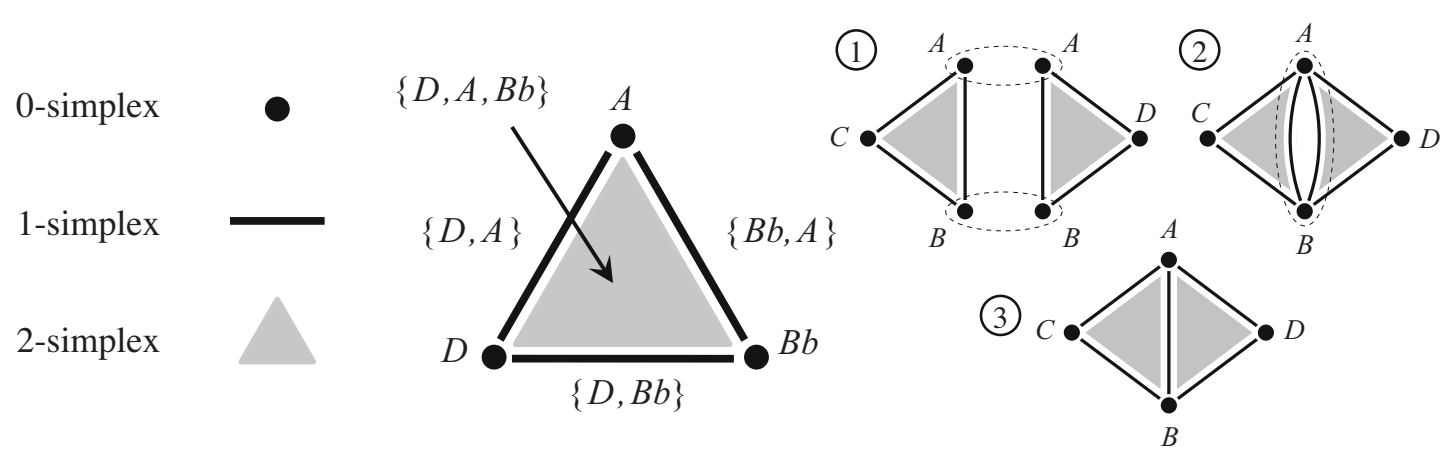

Fig. 1. On the left, three simplices. In the center, a 2-simplex and its boundary, representing the chord $\{D, A, B b\}$ and all two-note chords and notes included on it. On the right, the identification of boundaries illustrating the self-assembly process.

A result of this method is that a given pitch class set cannot be represented more than once in the simplicial complex. If the chords are ordered in the collection, this order will not be represented. For example, if the collection is a sequence of chords played successively, each chord will be represented but not their position in the sequence. We thus represent a temporal chord sequence by a static object, in the same way a photographer would catch a moving object by letting open the shutter of his camera. Different temporal chord sequences can be represented by topologically identical structure. This abstraction enables classifications of musical sequence based on topological criteria.

Chopin Prelude no. 4, Op. 28. Figure 2 shows the simplicial complex resulting from the assembly of the fifteen first chords of Chopin's Prelude Op.28, no. 4. The complex exhibits neighborhoods between chords but does not give any information about how these chords are ordered in the Prelude. A remarkable fact of this ordering is that only one note is different between two consecutive 


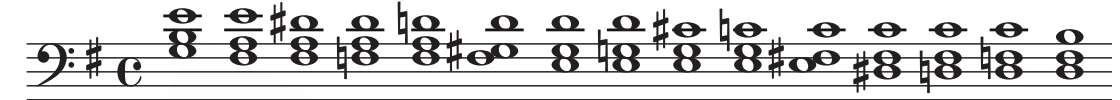
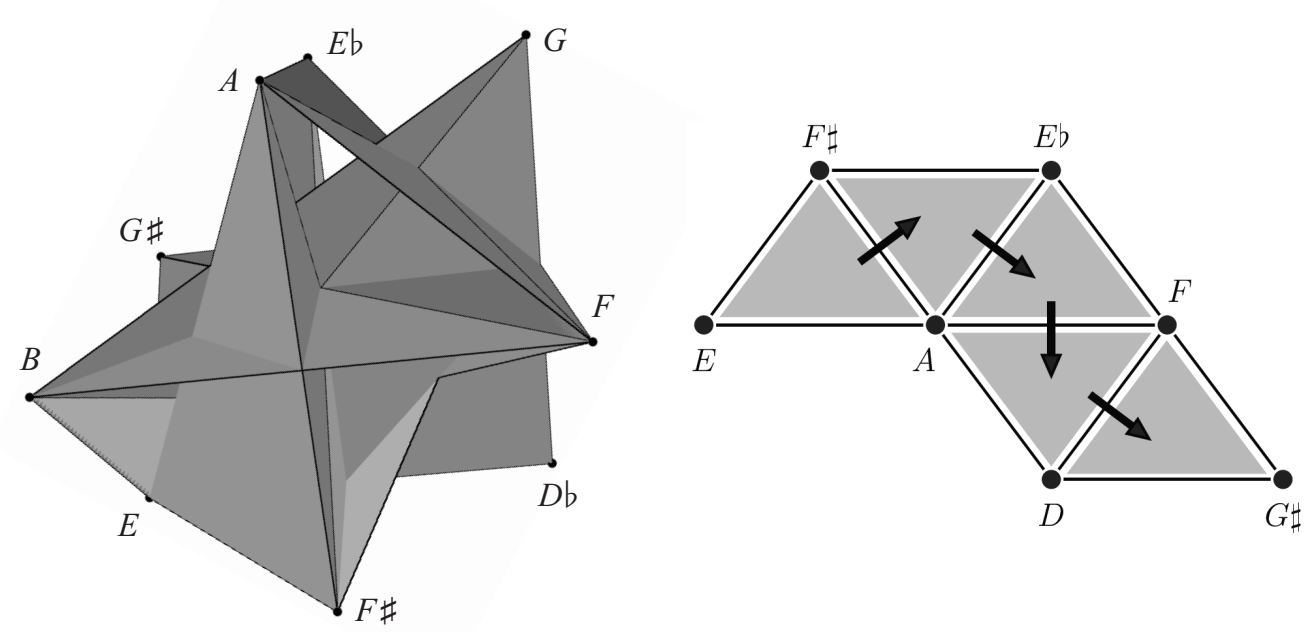

Fig. 2. Fifteen first chords of Chopin's Prelude Op. 28, no. 4. On the left its simplicial representation. On the right, a path represents the order of chords in a region of the complex.

chords. This property holds on for fourteen chords starting from the second one. Being composed of three-note chords, such a progression corresponds to a $(2,1)$-path in the associated simplicial complex: such a path is composed of 2 -simplices (the chords) connected by 1-simplices (the two common notes). This path is partially presented by black arrows for the five first chords in Figure 2. We have enumerated all the possible $(2,1)$-paths with length fourteen. It is interesting to note that there exist exactly 120 possible paths. Finally, among all these possibilities, the original order used in the Prelude is the one with the smallest distance between chords in terms of pitch motion. Indeed, the interval characterizing the moving note in two consecutive chords is a semitone for all transitions. This example illustrates the topological translation of a well-known compositional strategy called parsimonious voice leading.

\subsection{Chord Classes}

We now represent with the same process a set of chords, not organized in time, but defined by a specific property from a theoretical point of view. In the first examples, the self assembly process has been applied to chords associated with degrees of a scale thus offering a representation of a tonality [10]. The simplicial complex made from the triads of the diatonic scale is Mazzola's Möbius strip [11]. When assembling tetrahedra representing the seventh chords, the resulting complex is a toroid (the volume bounded by a torus).

An other way to categorize chords is to define equivalence classes. In this context, algebraic methods constitute useful and elegant tools for the classification [14]. 
Equivalence classes of chords can indeed be formalized as orbits under some group action. Transposition classes are determined by the action of the cyclic group $\mathbb{Z}_{N}$ on itself. Moreover, a set of chords equivalent up to transposition and inversion is specified as an orbit under the action of the dihedral group $D_{N}$ on the subsets of $\mathbb{Z}_{N}$. Other classifications can be established from the action of the group of affine transformations $A f f_{N}[12,11]$ and the symmetric group $S_{N}$ [13]. In the following, we particularly investigate simplicial complexes resulting from assembly of chords belonging to the same orbit under the action of $D_{12}$ on $\mathbb{Z}_{12}$ because it involves two musically relevant properties. Firstly, the orbits are equivalent to the 224 Forte classes [14]. Second is that their representations can be related to generalized Tonnetze. Nevertheless, the generic aspect of our approach makes possible the simplicial representation of equivalence classes under the action of any other group (the so-called paradigmatic classification).

$\boldsymbol{D}_{\boldsymbol{N}}$ Action on $\mathbb{Z}_{\boldsymbol{N}}$. In the case of the action of the dihedral group, orbits can be identified by an intervallic structure shared by all the chords of the orbit (and only these chords) [14]. This representation should not be confused with the interval vector. The intervallic structure represents a pitch class set by a series of consecutive intervals that always add up to $N$. These intervals are given by a list up to circular permutation and retrograde, which means up to transposition and inversion.

Let $\mathrm{X}$ be an interval structure. We write $\mathcal{C}(X)$ for the simplicial complex resulting from the assembly of simplices representing chords sharing the interval structure X.

Contrary to Forte names, the intervallic structure notation gives enough information to define all chords of the set class without having to refer to a list. Note that the congruence $N$ of the system do not need to be specified in the notation since it can be computed by summing elements of the intervallic structure. For instance $\mathcal{C}(4,3,3,2)$ and $\mathcal{C}(3,4,5)$, the simplicial complexes built by assembling minor and major chords, belong to the chromatic system $\mathbb{Z}_{12} \cdot \mathcal{C}(2,2,3)$, belongs to a heptatonic system $\mathbb{Z}_{7}$.

Chromatic Scale. Catanzaro investigates in [15] properties of simplicial complexes made from 2-simplices associated with transpositionnally and inversionnally related three-note chords in $\mathbb{Z}_{12}$. The 12 complexes represent the 12 different orbits of three-note chords under the action of $D_{12}$ on $\mathbb{Z}_{12}$. The 12 orbits correspond to the 12 Forte classes of size 3. Among the resulting complexes, the most frequent topology appears to be the torus. Thanks to the chord simplicial representation and self-assembly process described in section 2 , we extend this approach to investigate simplicial complexes representing all the other 212 (there are 224 orbits of subsets of $\mathbb{Z}_{12}$ under the action of $D_{12}$ ) orbits of $n$-note chords under this group action, with $0 \leqslant n \leqslant 12$. Chords are represented as simplices as described in section 3. Chords grouped together in the same orbit have the same cardinality. For this reason, they are represented by simplices of the same dimension. Highest dimensional simplices inside simplicial complexes representing orbits of size $n$ are $(n-1)$-simplices, which represent the $n$-note chords of the 
orbit. For example, the orbit including major and minor chords is represented by a complex resulting from the assembly of the 242 -simplices associated with all minor and major chords. Complexes of $n$-note chords are $(n-1)$-complexes. For $n=4$, they are built by gluing 3 -simplices which are tetrahedrons. Most orbits have 24 distinct forms (the order of $D_{12}$ ). The self-assembly process will then involve 24 different simplices. For example, the complex representing the set class including seventh and half-diminished seventh chords, is composed of 24 tetrahedra. Represented in three dimensions, these tetrahedra cross each other, making it hard to visualize the corresponding complex. Orbits that have fewer than 24 distinct forms are said to be symmetrical. This happens when some transposition or inversion corresponds to the identity transformation. These complexes present different topologies which can be effectively built and studied using the MGS programming language.

Heptatonic Scale. We consider here the action of $D_{7}$ on $\mathbb{Z}_{7}$. Simplicial complexes built from chords belonging to a heptatonic scale are interesting since they offer spatial representations of some tonalities. Mazzola's Möbius strip is an example of representation of one particular heptatonic scale, the diatonic scale. It is obtained by assembling chords whose intervallic structure in $\mathbb{Z}_{7}$ is $[2,2,3]$. The assembly of 3 -note chords of interval structure $[1,2,4]$ produces a 2 -dimensional simplicial complex in which all 0 -simplices (i.e. vertices) are neighbors.

Cayley Graphs and Generalized Tonnetze. Let $S$ be the 1-skeleton of a chordbased simplicial complex built from a set of chords equivalent up to inversion and transposition. $S$ is a graph composed by vertices representing all the pitch classes, and edges representing 2-note chords associated with a particular interval. If a pitch class is connected to an edge associated with an interval $i$ (for example a minor third), it is easy to see that, thanks to the transposition operation of the dihedral group, every other pitch class in $S$ will be connected to an edge associated with $i$ as well. As a consequence, the neighborhood of each pitch class of $S$ can be defined by the same set of intervals $J$. For this reason, $S$ can be related to a generalized Tonnetz in which pitch classes are neighboring according to a particular set of intervals. Moreover, by considering $J$ as a generating set of a subgroup of intervals $I$, one can consider the Cayley graph $\operatorname{Cay}(I, J)$ associated with the group presentation $\langle J \mid R\rangle$ where $R$ is the set of relations linking the elements of $J$. Cay $(I, J)$ can be related to $S$.

A simplicial complex $\mathcal{C}(X)$ is thus related to a particular generalized Tonnetz defined by intervals contained in chords having the interval structure $X$. But a generalized Tonnetz can be related to several simplicial complexes. For example $\mathcal{C}(1,3,4,4), \mathcal{C}(1,3,5,3)$ and $\mathcal{C}(1,3,1,3,1,3)$ are all related to the same generalized Tonnetz in which two pitch classes are neighbor if they are separated by an interval class in $\{1,3,4,5\}$. For $N=12$, among the 64 generalized Tonnetze computed by enumerating all possible interval class sets, only 30 correspond to a 1-skeleton of a chord-based simplicial complex. 


\section{Using Chord-Based Simplicial Complexes for Computational Music Analysis}

In this section, we present methods to visualize musical sequences in chord-based complexes. These methods are implemented in Hexachord, a computer-aided music analysis environment.

\subsection{Sequence Visualization in Unfolded Chord-Based Complexes}

Unfolding Simplicial Complexes. As previously mentioned, simplicial complexes are often hard to visualize. Depending on their topological properties, simplicial complexes can be unfolded as infinite planes to make their visualization easier. The major difference between a simplicial complex and its unfolded representation is that in the former, pitch classes are represented once, and in the latter, by an infinite number of occurrences. Moreover, the graph corresponding to the unfolding can be embedded in the Euclidean space such that parallel 1-simplices relate to the same interval class. An essential advantage of this unfolding is that it preserves the neighborhood between elements. The neighbors in the unfolded representation are the neighbors in the original representation. The unfolded representation is built as follows: one chord of the set class is represented by its simplex. Then, 1-simplices (i.e., edges) are extended as infinite lines. The interval labelling the edge is assigned to the line and all its parallels. Pitch classes and chords are organized and repeated infinitely following the lines by respecting the assigned intervals. By considering 1-skeletons of unfolded $\mathcal{C}(3,4,5)$ and $\mathcal{C}(2,4,3,3)$ (Figure 3$)$, one gets, respectively, the neo-Riemannian Tonnetz [2] and the Gollin 3D Tonnetz [16].

Chord classes complexes resulting from the assembly of $n$-note chords are unfolded as $(n-1)$-dimensional infinite spaces. $\mathcal{C}(5,7)$ is unfolded as an infinite line, $\mathcal{C}(3,4,5)$ and $\mathcal{C}(2,2,3)$ as infinite triangular tessellations. Note that $n$-simplices don't systematically tessellate the $n$-dimensional Euclidean space. For example, 2 -simplices (triangles) tessellate the $2 \mathrm{D}$ plane but 3 -simplices (tetrahedra) do not tessellate the 3D space. For this reason, the 3D unfolded representation of complexes as $\mathcal{C}(2,4,3,3)$ contains some holes.

Visualization in Hexachord. Hexachord ${ }^{1}$ is a computer-aided music analysis environment, based on the previous simplicial representations. A first functionality is the visualization of the sequence of chords in a midi file inside some simplicial complexes related to generalized Tonnetze.

Hexachord offers the visualization of musical sequences in unfolded representations of simplicial complexes built from three-note chords. As previously mentioned, these unfoldings are infinite $2 \mathrm{D}$ triangular tessellations. ${ }^{2}$ When a

\footnotetext{
${ }^{1}$ Presentation videos available at http://vimeo.com/38102171

${ }^{2} 3 \mathrm{D}$ representation of unfolded tetrahedral spaces composed by 4 -note chords in Hexachord is currently under development.
} 


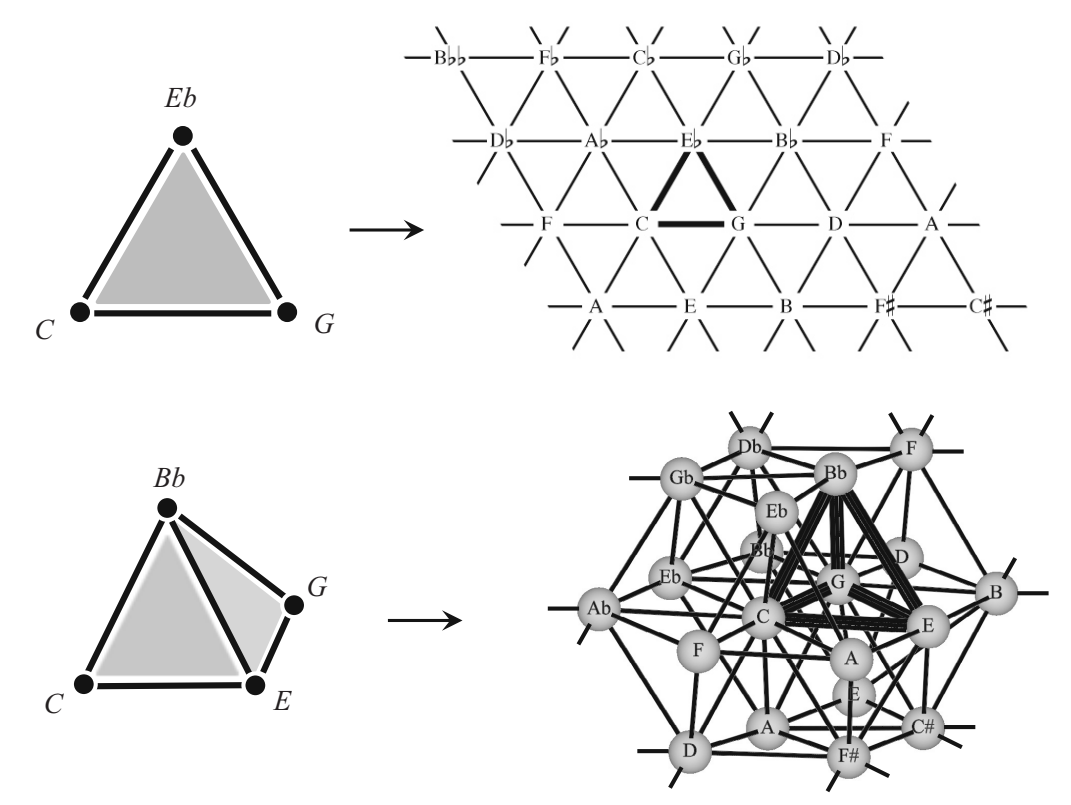

Fig. 3. On the top, the unfolding process is applied to $\mathcal{C}(3,4,5)$ by extending $\mathrm{C}$ Major 1-simplices to infinite lines on the plane. At the bottom, unfolding process is applied to $\mathcal{C}(2,4,3,3)$ in the $3 \mathrm{D}$ space.

midi file is read, cells representing played pitch classes and chords are filled in real-time in light yellow (See Figure 4). Their remanence (in an alternate color) can be adjusted to render the motion as a path in the complex.

We mentioned in the previous section that pitch classes and chords occur at multiple locations in unfolded representations. As a consequence, multiple paths can be chosen to represent the same sequence of chords. Figure 4 illustrates this phenomenon by a simple example. The sequence representation at the top shows that the transition from $C$ to $G$ can be interpreted as different motions in $\mathcal{C}(1,2,9)$, for the reason that this region of the unfolded representation of the complex includes two occurrences of the pitch class $C$ and three of $G$.

The user can thus choose to illuminate every location representing a played element, or just one in order to observe motions locally, as shown at the bottom of Figure 4. Locations are chosen by following both static and dynamic criteria. The static criterion requires that when several elements are played together, the filled cells must be as close as possible from each other. In other words, the region containing filled cells must be as compact as possible. This criterion allows to interpret pitch class sets as geometrical shapes. The dynamic criterion requires the evolution from a set of elements to another one to be represented by the smallest possible motion. This second criterion facilitates the interpretation of a motion as a geometrical transformation. 


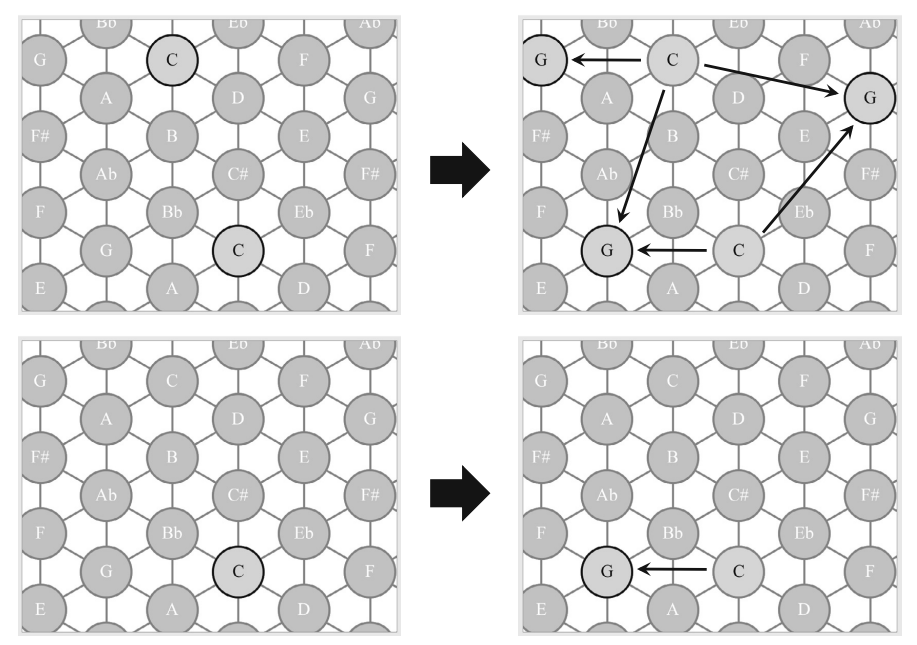

Fig. 4. Visualization with Hexachord of the pitch sequence $[C, G]$ in a region of the unfolded representation of $\mathcal{C}(1,2,9)$. On the top each cell labelled by the played pitch is illuminated. On the bottom, pitch classes are illuminated in a single location. Arrows represent possible interpretations of the motion during the sequence.

\subsection{Introduction of the Compliance}

We call compliance the capacity of a space to reveal the regularity of an object and/or its evolution, when represented in it. Informally, a regularity is something which cannot be interpreted as randomness.

In the context of our musical study, we will consider only a limited set of spaces. Objects refer to pitch class sets. Their evolutions are interpreted as pitch class sequences and pitch class set sequences. Spaces are simplicial complexes whose 1-skeletons are generalized pitch class Tonnetze. These complexes are built by assembly of simplices representing pitch class sets related by transposition and inversion as described in the previous section. Any possible pitch class set can be used to build a particular simplicial complex among the 224. Its 1-skeleton is one of the 30 generalized Tonnetze described in section 3. Before giving a more precise definition of the compliance, let's look at an example motivating our approach.

Chord Sequence in $\mathcal{C}(\mathbf{3}, \mathbf{4 , 5})$. The interpretation of measures 143 to 176 of the second movement of Beethoven's ninth Symphony has been frequently studied as a succession of neo-Riemannian operations $R$ and $L[2,17]$.

The representation of this sequence in the Tonnetz reveals a geometric regularity due to interval properties shared by the space and the chord sequence [2]. This regularity can be noticed in $\mathcal{C}(3,4,5)$ due to the deep relation, highlighted in the previous section, between this complex and the original Tonnetz. Figure 6 compares representations of this chord sequence in $\mathcal{C}(3,4,5)$ and $\mathcal{C}(1,2,9)$. The spatial regularity emerging in $\mathcal{C}(3,4,5)$ illustrates the compliance of this space with this chord sequence. We see two main reasons for this regularity: 


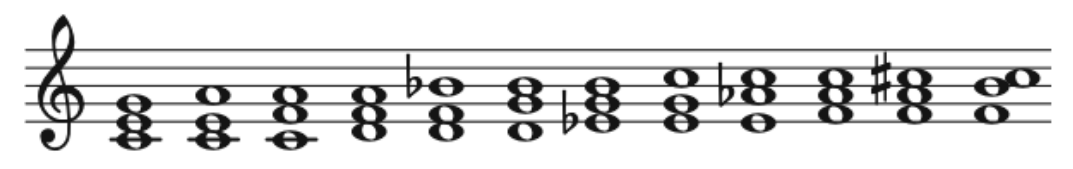

Fig. 5. Chord sequence extracted from Beethoven's $9^{\text {th }}$ Symphony

1. Three-note chords used in this sequence are represented by 2 -simplices thus by compact objects.

2. The regular alternation between the neo-Riemannian operations $L$ and $R$ represents the sequence as following a straight trajectory.

The first property is static, the second one is dynamic. In the following we investigate the static property by proposing a method to measure compactness of pitch class sets in simplicial complexes. Of course, compactness is not the only property to take into consideration when estimating the regularity of musical objects representations in these complexes. Nevertheless, it makes an interesting first indication.
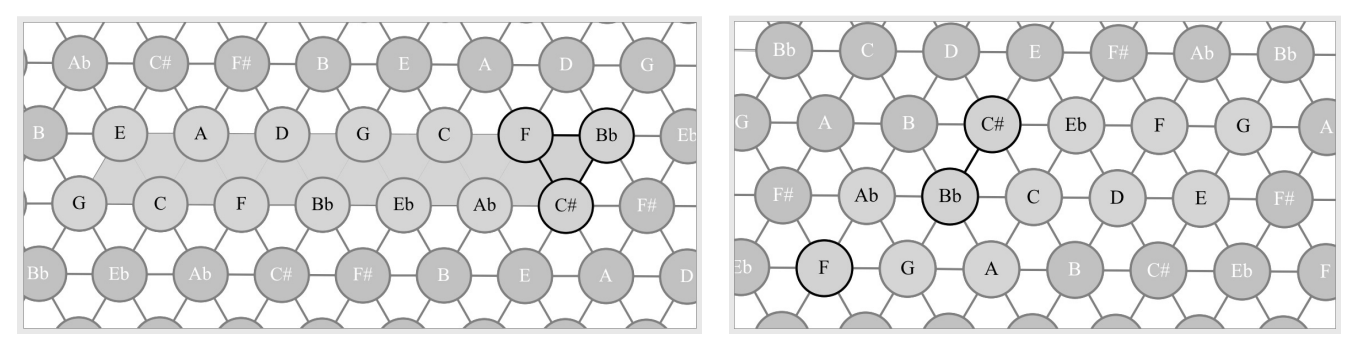

Fig. 6. Evolution of the chord sequence extracted from Beethoven's $9^{\text {th }}$ Symphony in unfolded representation of $\mathcal{C}(3,4,5)$ (on the left) and $\mathcal{C}(1,2,9)$ (on the right)

\subsection{Measure of Compactness}

As a first example of compliance, we propose here a method to calculate compactness of a pitch class set in a simplicial complex by a sub-complex. We define the compactness of a simplicial complex $A$ at the dimension $m$ by

$$
m \text {-compactness }(A)=\frac{f_{m+1}(A)}{\left(\begin{array}{c}
f_{1}(A) \\
m+1
\end{array}\right)}
$$

In other words, the $m$-compactness compares the number of $m$-simplices the complex has, with the number of $m$-simplices it could maximally have given its number of vertices. The values of $m$-compactness lie in the real interval $[0,1]$.

This definition of compactness depends on the dimension $m$. For example, the 1-skeleton of a tetrahedron (composed by 4 vertices and 6 edges) has a 1compactness equal to 1 and a 2-compactness equal to 0 . Naturally, these different forms of compactness are related. Especially, the existence of high dimensional simplices induces compactness of the lower levels. For example, if a complex 
includes a 3-simplex (a tetrahedron), it includes by definition its four vertices too, which are all neighbor and thus compact. Thus, compactness at a high level induces compactness at lower levels. However, compactness at a low level does not necessarily induce compactness at higher levels. For example, four neighbor vertices don't necessarily surround a 3-simplex.

The pitch class set $A$ is represented in $\mathcal{C}$ by a sub-complex $\mathcal{C}_{A}$ of $\mathcal{C} . \mathcal{C}_{A}$ is composed by all simplices in $\mathcal{C}$ representing an element included in $A$ (pitch classes, two-note chords, etc.). In other words, $\mathcal{C}_{A}$ is the intersection between $\mathcal{S}_{A}$ (the simplicial representation of $A$ as defined in Section 3) and $\mathcal{C}$. The topological aspect of $\mathcal{C}_{A}$ thus results from the constitution of $\mathcal{C}$. Figure 7 shows the intersection between chord $(C, E, G, B b)$ and $\mathcal{C}(3,4,5)$ and between chord $(C, G, B)$ and $\mathcal{C}(2,3,5)$.
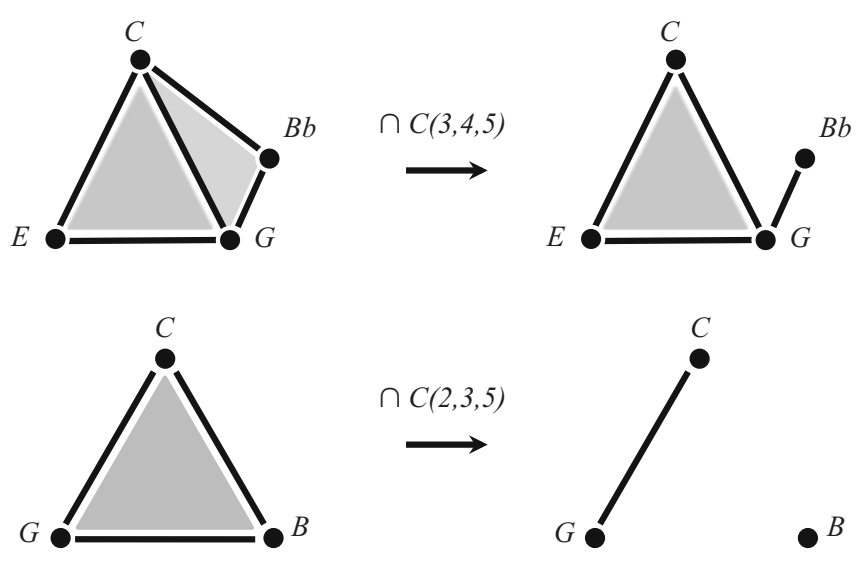

Fig. 7. On the left, the simplicial representations of chords $(C, E, G, B b)$ (top) and $(C, G, B)$ (bottom) and on the right their intersection respectively with $C(3,4,5)$ and $C(2,3,5)$

The compliance relates the $m$-compactness of an $n$-sized pitch class set $A$ in a complex $\mathcal{C}$ by the formula:

$$
\frac{f_{m+1}\left(\mathcal{S}_{A} \cap \mathcal{C}\right)}{\left(\begin{array}{c}
n \\
m+1
\end{array}\right)}
$$

where $\mathcal{S}_{A}$ is the simplicial representation of $A$.

Statistics on Chord Sequences. A musical sequence can be seen as a sequence $\left\{A_{i}\right\}_{i \in \llbracket 0, s i z e \rrbracket}$ of pitch class sets. Each time a new pitch class is played, or a played pitch class is stopped, a new set is created and concatenated in the sequence. The duration of a pitch class set $A_{i}$ is noted $d_{i}$ and the total duration of the sequence is noted $D=\sum_{i=0}^{\text {size }} d_{i}$. We can thus compute the $m$-compactness of a complex $\mathcal{C}$ with the sequence $\left\{A_{i}\right\}$ by computing 


$$
\frac{1}{D} \times \sum_{i=0}^{\text {size }}\left[\frac{f_{m+1}\left(\mathcal{S}_{A_{i}} \cap \mathcal{C}\right)}{\left(\begin{array}{c}
f_{1}\left(\mathcal{S}_{A_{i}}\right) \\
m+1
\end{array}\right)} \times d_{i}\right]
$$

Some Applications. This approach can be useful in musical analysis since it enables classification. For example, one can be interested in finding most compliant spaces with a chord progression, a whole piece or a corpus related to a style or an author. Figure 8 shows the average 2-compactness of the twelve complexes built from three-note chords for four jazz standards. Each red bar represents the average compactness of the piece in a particular complex. Some similarities between these histograms seem to represent common practices in jazz. In particular, most 2-compact spaces are for each piece $\mathcal{C}(2,3,7), \mathcal{C}(2,4,6)$ and $\mathcal{C}(3,4,5)$.
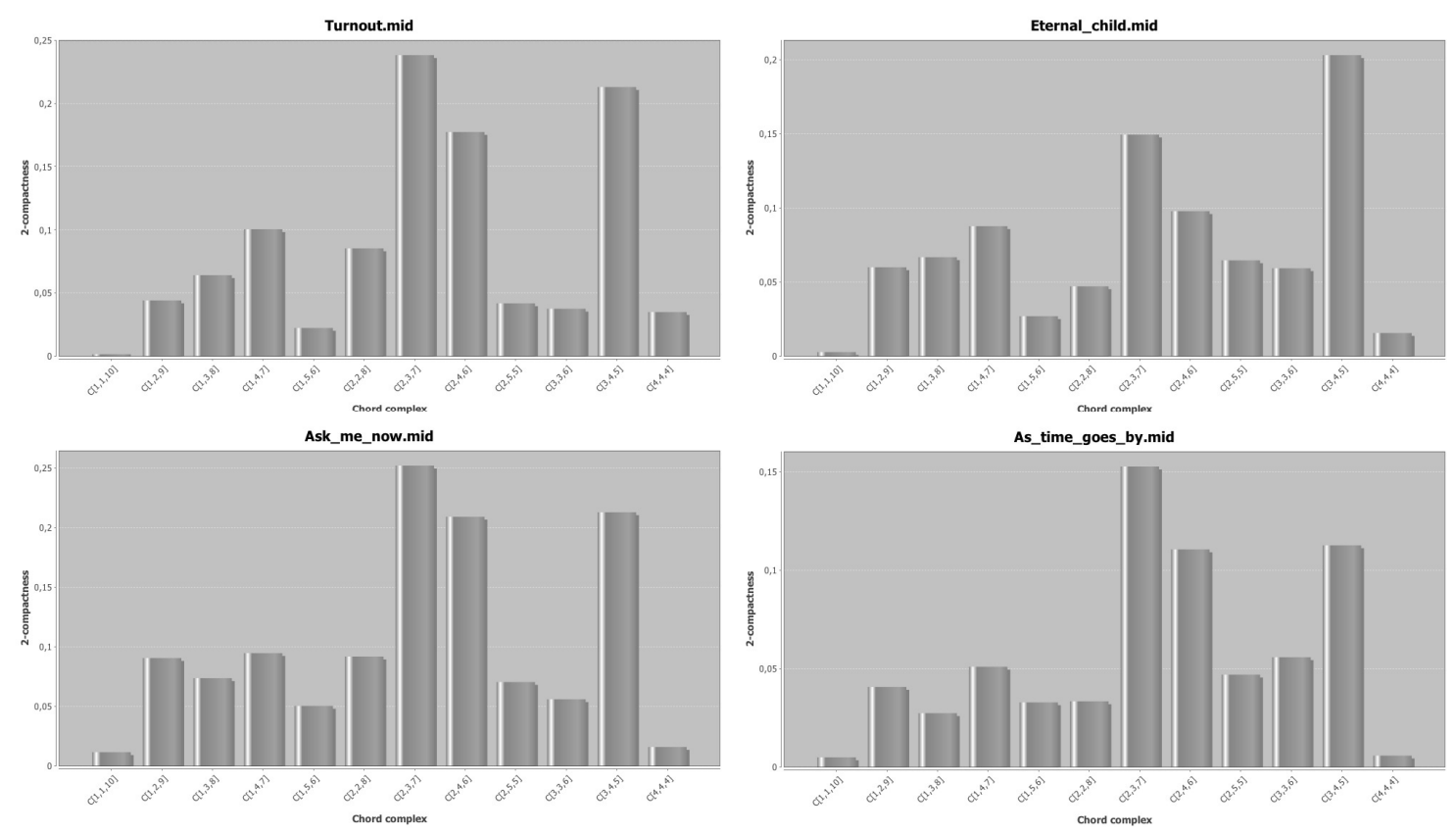

Fig. 8. Average compliance of the twelve complexes built from three-note chords with the standards Turn Out The Stars of Bill Evans (top left), Eternal Child of Chick Corea (top right), Ask Me Now of Thelonious Monk (down left) and As Time Goes By of Art Tatum (down right)

Figure 9 shows the same measure on the whole second movement of Beethoven's ninth Symphony and Schönberg's Klavierstücke Op. 19, No. 6. The difference of style is here expressed by the need to switch from $\mathcal{C}(3,4,5)$ to $\mathcal{C}(2,3,7)$ to visualize compact three-note chords.

Another possible application is harmonization by spatial criteria. An extra pitch class is added to a pitch class set when it maximizes the compliance of a given space with this set. 

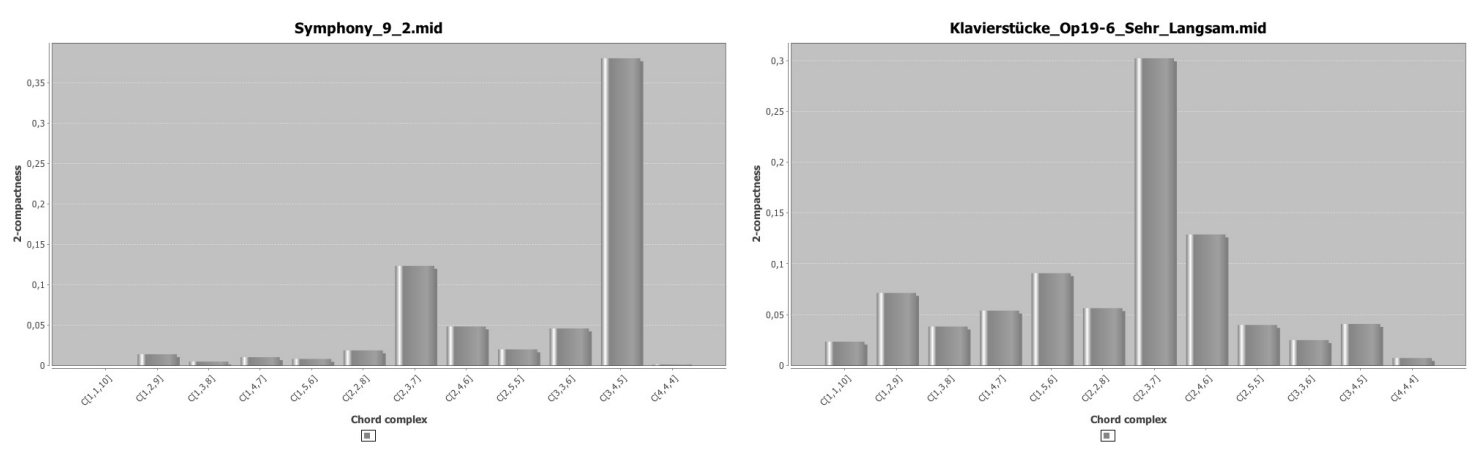

Fig. 9. Average compliance of the 12 complexes built from three-note chords with second movement of Beethoven $9^{\text {th }}$ Symphony (left) and Schoenberg Klavierstücke Op. 19 , No. 6

\section{Conclusion and Future Works}

Simplicial representation of chords is a powerful tool to analyse chord sequences and musical style. However, this analysis could be more fruitful by considering a more complete catalog of chord based complexes. We plan to investigate particularly complexes built from equivalence classes described by Mazzola [11] and Julio Estrada [13]. Moreover, the research of a compliant space with a musical piece rarely ends in a unique complex. The comparison of complex compliance over time aids in the harmonic segmentation of the piece. A study of the successive most compliant complexes during a piece gives interesting information about composers' practices.

Finally, as illustrated for parsimonious voice leading in section 3.1, we are interested in translating compositional strategies into topological rules that specify paths on chord complexes.

Acknowledgments. The authors are very grateful to the REPMUS team at IRCAM, to Jean-Marc Chouvel and Mikhail Malt for endless fruitful discussions. This research is supported in part by the IRCAM and the University Paris EstCréteil Val de Marne.

\section{References}

1. Chew, E.: The Spiral Array: An Algorithm for Determining Key Boundaries. In: Anagnostopoulou, C., Ferrand, M., Smaill, A. (eds.) ICMAI 2002. LNCS (LNAI), vol. 2445, pp. 18-31. Springer, Heidelberg (2002)

2. Cohn, R.: Neo-Riemannian Operations, Parsimonious Trichords, and their "Tonnetz" Representations. Journal of Music Theory 41(1), 1-66 (1997)

3. Callender, C., Quinn, I., Tymoczko, D.: Generalized Voice-Leading Spaces. Science 320(5874), 346 (2008)

4. Tymoczko, D.: The Geometry of Musical Chords. Science 313(5783), 72 (2006)

5. Giavitto, J.L., Michel, O.: MGS: a Rule-Based Programming Language for Complex Objects and Collections. In: van den Brand, M., Verma, R. (eds.) Electronic Notes in Theoretical Computer Science, vol. 59. Elsevier, Amsterdam (2001) 
6. Giavitto, J.L.: Topological Collections, Transformations and their Application to the Modeling and the Simulation of Dynamical Systems. In: Nieuwenhuis, R. (ed.) RTA 2003. LNCS, vol. 2706, pp. 208-233. Springer, Heidelberg (2003)

7. Munkres, J.: Elements of Algebraic Topology. Addison-Wesley (1984)

8. Spicher, A., Michel, O., Giavitto, J.-L.: Declarative Mesh Subdivision Using Topological Rewriting in mgs. In: Ehrig, H., Rensink, A., Rozenberg, G., Schürr, A. (eds.) ICGT 2010. LNCS, vol. 6372, pp. 298-313. Springer, Heidelberg (2010)

9. Giavitto, J.L., Spicher, A.: Simulation Of Self-Assembly Processes Using Abstract Reduction Systems. In: Krasnogor, N., Gustafson, S., Pelta, D.A., Verdegay, J.L. (eds.) Systems Self-Assembly: Multidisciplinary Snapshots, pp. 199-223. Elsevier, Amsterdam (2008)

10. Bigo, L., Giavitto, J., Spicher, A.: Building topological spaces for musical objects. In: Agon, C., Andreatta, M., Assayag, G., Amiot, E., Bresson, J., Mandereau, J. (eds.) MCM 2011. LNCS, vol. 6726, pp. 13-28. Springer, Heidelberg (2011)

11. Mazzola, G., et al.: The Topos of Music: Geometric Logic of Concepts. In: Theory, and Performance. Birkhäuser (2002)

12. Morris, R.: Composition with Pitch Classes: a Theory of Compositional Design. Yale University Press, New Haven (1987)

13. Estrada, J.: La teoría d1, MúSIIC-Win y algunas aplicaciones al análisis musical: Seis piezas para piano, de Arnold Schoenberg. In: Lluis-Puebla, E., AgustínAquinas, O. (eds.) Memoirs of the Fourth International Seminar on Mathematical Music Theory, Huatulco (2011)

14. Andreatta, M., Agon, C.: Implementing Algebraic Methods in openmusic. In: Proceedings of the International Computer Music Conference, Singapore (2003)

15. Catanzaro, M.: Generalized Tonnetze. Journal of Mathematics and Music 5(2), 117-139 (2011)

16. Gollin, E.: Some Aspects of Three-Dimensional "Tonnetze". Journal of Music Theory 42(2), 195-206 (1998)

17. Albini, G., Antonini, S.: Hamiltonian Cycles in the Topological Dual of the Tonnetz. In: Chew, E., Childs, A., Chuan, C.-H. (eds.) MCM 2009. CCIS, vol. 38, pp. 1-10. Springer, Heidelberg (2009) 